\title{
OPEN Signatures of natural selection and ethnic-specific prevalence of NPC1 pathogenic mutations contributing to obesity and Niemann-Pick disease type C1
}

\author{
Andreea Chiorean $\mathbb{1}^{1}$, William S. Garver $\mathbb{1}^{2}$ \& David Meyre $\mathbb{1}^{1,3 \bowtie}$
}

While homozygous pathogenic mutations in the NPC1 gene cause Niemann-Pick type C1 disease, heterozygous mutations cause highly-penetrant obesity. We aimed to investigate the prevalence of NPC1 mutations and their signatures of natural selection in 122,678 exome sequenced participants from six ethnic groups in the Genome Aggregation Database. Pathogenic missense coding mutations were identified by in silico tools and the ClinVar database. Signatures of natural selection were assessed by the probability of NPC1 being loss-of-function mutation intolerant and Z-scores of observed/expected synonymous and non-synonymous mutation ratios. There was no evidence of negative selection observed for synonymous, non-synonymous and loss-of-function mutations. However, there were significant ethnic differences in the prevalence of heterozygous pathogenic NPC1 mutations ranging from $0.56 \%$ in Ashkenazi Jewish to $3.26 \%$ in African/African Americans (5.8-fold difference). Four homozygous carriers of pathogenic NPC1 mutations were also identified, belonging to the South Asian population. In conclusion, NPC1 mutations are consistent with a model of balanced selection, where heterozygotes and homozygotes have higher and lower reproductive fitness, respectively. Therefore, NPC1 heterozygous mutations may account for a substantial and ethnicdependent percentage of obesity in the general population, while NPC1 homozygous mutations may be frequent in the South Asian populations and warrants more investigation.

The prevalence of global adult obesity has almost tripled since 1975 to reach 650 million in 2016 according to the World Health Organization. In the past four decades, the number of obese children aged five to 19 has increased tenfold ${ }^{1}$. Obesity is associated with the development of multiple comorbidities ${ }^{2}$. For instance, obesity accounts for $24.3 \%$ and $3.6 \%$ of the risks for cardiovascular disease and cancer, respectively, the two leading causes of death worldwide ${ }^{3,4}$. Despite several therapeutic modalities (lifestyle and behavioral modifications, medication, bariatric surgery), obesity remains difficult to treat ${ }^{5}$, highlighting the need for new knowledge to predict, prevent, and manage this illness ${ }^{6,7}$. It is well known that obesity results from a complex interplay between biological factors, including genetics, and the environment ${ }^{6,8}$. Twin, family and population studies estimate that $40-75 \%$ of body mass index (BMI) variation is explained by genetic determinants ${ }^{9,10}$. Multiple genes responsible for syndromic and non-syndromic monogenic, oligogenic and polygenic forms of obesity have been recently identified in various ethnic groups $s^{6,9,11,12}$, including the human Niemann-Pick C1 (NPC1) gene ${ }^{13,14}$.

The NPC1 protein mediates the transport of low-density lipoprotein-derived cholesterol and fatty acids from late endosomes/lysosomes into the cytoplasm and other cellular compartments, regulates feedback inhibition of the sterol regulatory binding protein (SREBP) and feedforward activation of the liver X receptor (LXR) pathways $^{15}$. Homozygous or heterozygous compound loss-of-function (LOF) mutations in NPC1 cause NiemannPick type C1 (NPC1) disease, an autosomal recessive lipid storage disorder ${ }^{16}$ with the estimated incidence of $1 / 92,000^{17}$. Despite a large spectrum of clinical phenotypes, most patients with NPC1 disease have a life span between 10 and 25 years due to complications resulting from liver failure and neurological degeneration ${ }^{16}$. Similar

${ }^{1}$ Department of Health Research Methods, Evidence, and Impact, McMaster University, 1280 Main Street West, Michael G. DeGroote Centre for Learning and Discovery Room 3205, Hamilton, ON L8S 4K1, Canada. ${ }^{2}$ Department of Chemistry and Chemical Biology, University of New Mexico, Albuquerque, NM, USA. ${ }^{3}$ Department of Pathology and Molecular Medicine, McMaster University, Hamilton, ON, Canada. ${ }^{\varpi}$ email: meyred@mcmaster.ca 


\begin{tabular}{|l|l|l|l|}
\hline Category & Expected No. variants & Observed No. variants & Constraint metrics \\
\hline Synonymous & 290.7 & 314 & $\begin{array}{l}\mathrm{Z}=-1.07 \\
\mathrm{o} / \mathrm{e}=1.08(0.98-1.19)\end{array}$ \\
\hline Missense & 719.4 & 637 & $\begin{array}{l}\mathrm{Z}=1.09 \\
\mathrm{o} / \mathrm{e}=0.89(0.83-0.94)\end{array}$ \\
\hline LOF & 62.2 & 21 & $\begin{array}{l}\mathrm{pLI}=0.00 \\
\mathrm{o} / \mathrm{e}=0.34(0.43-0.49)\end{array}$ \\
\hline
\end{tabular}

Table 1. GnomAD gene constraint for NPC1. o/e observed/expected score, $L O F$ loss of function, $p L I$ probability of being loss-of-function intolerant.

to humans, a multitude of different NPC1 mouse models have been characterized with NPC1 disease-associated phenotypes including time-dependent cholesterol accumulation in most cells/organs, hepatomegaly, shortened lifespan, and signs of progressive neurologic impairment ${ }^{18-20}$. In 2009, a genome-wide association study (GWAS) identified two common NPC1 non-synonymous single-nucleotide polymorphisms (SNPs) (rs1805081/H215R and $\mathrm{rs} 1805082 / \mathrm{I} 858 \mathrm{~V}$ ) in linkage disequilibrium that were significantly associated with polygenic morbid obesity in European adult populations but not in European children ${ }^{13}$. More recently, a genome-wide significant association was found between the correlated SNPs rs1805081/H215R, rs1788799/M642I, and rs1805082/I858V) and body mass index (BMI) in predominantly European adult populations ${ }^{21}$. Furthermore, men with rare heterozygous NPC1 LOF mutations who had children with NPC1 disease had a significantly higher BMI than noncarriers in an East Asian population ${ }^{14}$. Young patients of the same population carrying heterozygous NPC1 LOF mutations had a fivefold increase in the risk of obesity, although only the associations in men reached significance when the results were stratified by $\operatorname{sex}^{14}$. Similar sex-specific associations were found in NPC1 heterozygous knock-out $\left(\mathrm{NPC1}^{+/-}\right)$mice fed a high fat diet (HFD) with significant differences in body weight observed as the mice reached maturity ${ }^{14}$. Other studies also observed a latent weight gain in $N P C 1^{+1-}$ mice fed a HFD ${ }^{22-25}$, supporting the adult onset of obesity observed in human NPC1 variant carriers ${ }^{13,21}$. More recently, studies have reported that $\mathrm{NPC1}^{+/-}$mice fed a high-fat diet are physiologically characterized with increased liver glycolysis and lipogenesis, and decreased adipose lipolysis through impaired feedback inhibition of the sterol regulatory element binding protein-1 (SREBP-1) pathway. These metabolic disturbances lead to lipid accumulation in the liver and adipose tissue with resultant weight gain in $\mathrm{NPC1}^{+/-}$mice compared to $\mathrm{NPC1}^{+/+}$mice fed an identical diet, thereby confirming the gene-diet interaction ${ }^{23}$.

Since NPC1 patients are known to have low reproductive success due to premature death, NPC1 LOF homozygous/heterozygous compound mutations are prone to negative selection ${ }^{26,27}$. On the contrary and consistent with the 'thrifty genotype hypothesis' ${ }^{28}$, NPC1 LOF heterozygous carriers may have been positively selected for during historical periods of famine, considering their increased ability to store fat. This pattern of balancing selection with an heterozygous advantage has been previously described for the widely-accepted 'malaria hypothesis ${ }^{29,30}$. Individuals with homozygous mutations for sickle hemoglobin develop sickle cell anemia, a fatal disease if left untreated ${ }^{29}$, while heterozygotes have a protective advantage against the Plasmodium falciparum malaria infection ${ }^{30}$.

With respect to rare NPC1 mutations several important gaps of knowledge remain. Specifically, the ethnic specific distribution and signatures of natural selection signature of NPC1 rare mutations have not been described. Therefore, utilizing the Genome Aggregation Database (gnomAD), we assessed the prevalence and signatures of natural selection of NPC1 mutations in six ethnic groups (European, Ashkenazi Jewish, East Asian, South Asian, Latino, and African/African American).

\section{Results}

Signatures of natural selection for NPC1 mutations. NPC1 displayed a pLI value of 0 (21 observed versus 62.2 expected number of LOF mutations (Table 1). This signifies that the presence of haploinsufficient LOF mutations (nonsense, splice acceptor, splice donor, and frameshift) in this gene is well-tolerated. No significant enrichment or depletion of missense (637 observed versus 719.4 expected, $Z=1.09$ ) or synonymous (314 observed versus 290.7 expected, $Z=-1.07$ ) mutations was observed. Similarly, the observed/expected mutation ratio $90 \%$ confidence intervals for LOF, missense and non-synonymous mutations were not indicative of strong mutation intolerance (all 90\% confidence interval upper bound values $\geq 0.35$, Table 1 ).

Description of the NPC1 pathogenic mutations identified in gnomAD. We identified 414 distinct rare pathogenic mutations (minor allele frequency $<1 \%$ in all ethnic groups) in 1574 heterozygous and four homozygous carriers from six ethnic populations in gnom $\mathrm{AD}(\mathrm{N}=122,678)$ : 34 frameshift $(8.2 \%), 6$ splice acceptor (1.4\%), 6 splice donor (1.4\%), 13 stop gained (3.1\%), and 355 (85.7\%) missense mutations. Two of the missense mutations identified as non-pathogenic by SIFT/Polyphen were then identified as pathogenic on ClinVar and included in the analysis. Two-hundred ninety-two missense mutations were classified as non-pathogenic and were not further investigated. Six pathogenic mutations (five missense and one stop gained) were removed from the study because they were only present in the 'other' ethnicity group and nine mutations (six missense, two stop lost and two frameshift) with protein consequences not corresponding to isoform 1 were also excluded. More details on these mutations can be found in Supplementary Table 1. 


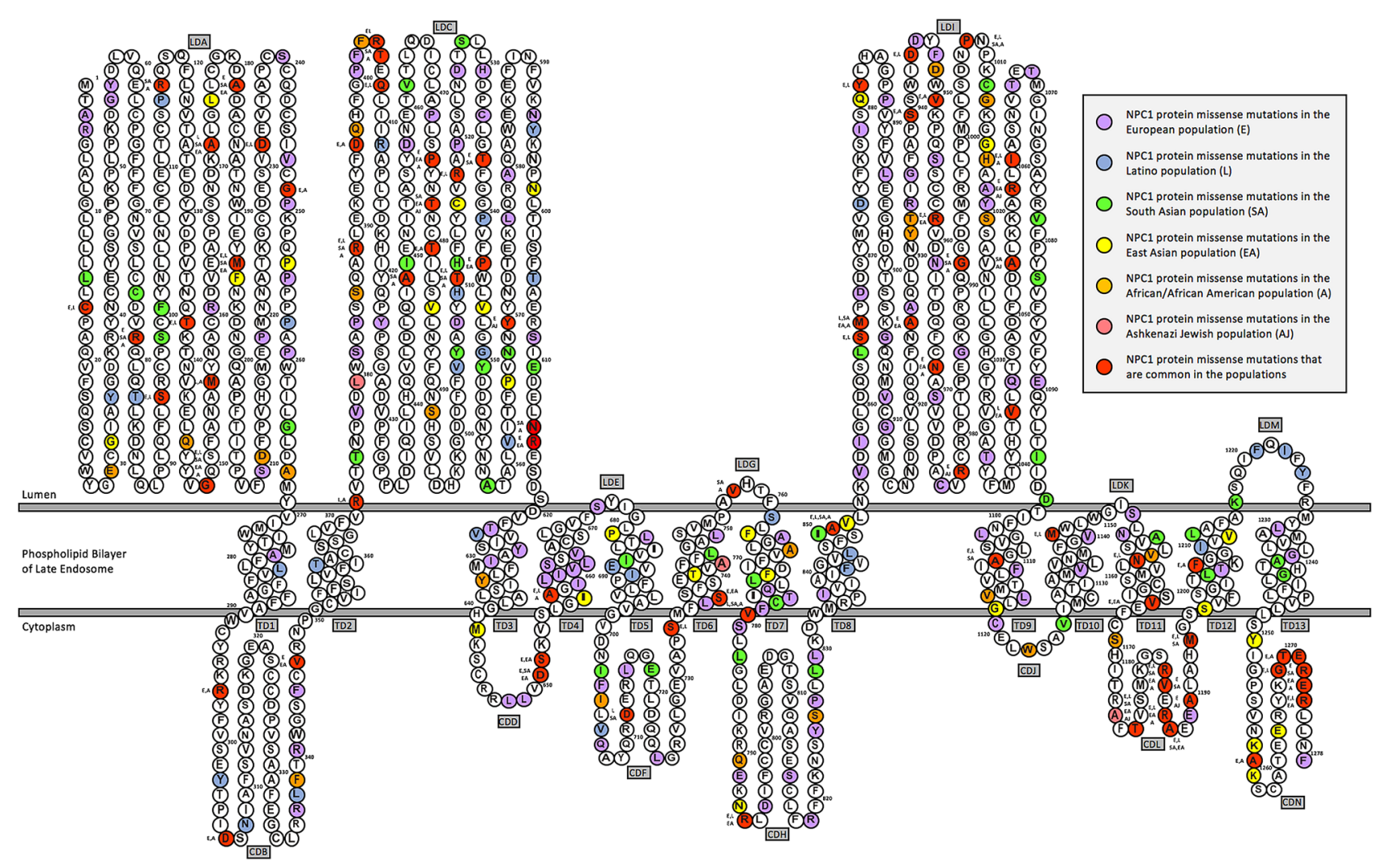

Figure 1. NPC1 protein model of coding pathogenic mutations of six ethnic groups from the gnomAD database. The first amino acid (methionine, Met, or M) positioned at the amino-terminus (left hand side of the figure) is numbered with a superscript on the upper right side of the symbol for methionine $\left(\mathrm{M}^{1}\right)$. The other NPC1 protein amino acids are similarly numbered with a superscript every ten amino acids to facilitate identification of all 1278 amino acids. The location for the 402 coding pathogenic mutations corresponding to ancestral amino acids are color-coded based on the six populations. The red color-coded ancestral amino acids represent locations where a missense mutation for the same or different derived amino acids is present in another population. To indicate these populations, the red color-coded ancestral amino acids are annotated on the middle left side using acronyms for the six populations: Europeans (E), Latino (L), South Asian (SA), East Asian (EA), African/African American (A), and Ashkenazi Jewish (AJ). NPC1 protein domains are designated as luminal domains (LD), cytoplasmic domains (CD), and transmembrane domains (TD) labeled alphanumerically starting from the NPC1 protein amino-terminus and ending at the NPC1 carboxyl-terminus. The sterol-sensing domain (SSD) composed of TD3-TD7.

The location of each pathogenic coding mutation is summarized in an NPC1 protein model (Fig. 1) with the luminal domains (LD), cytoplasmic domains (CD), and transmembrane domains (TD) labeled alphanumerically starting from the NPC1 protein amino-terminus and ending at the NPC1 carboxyl-terminus ${ }^{31}$. Pathogenic missense mutations were categorized by their location in the $27 \mathrm{NPC} 1$ protein domains and are available in Supplementary Table 2. The highest gross number of pathogenic missense mutations was associated with the LDC domain (73 mutations) known to facilitate binding of the NPC2 protein and assorted viruses, the LDI or cysteine-rich domain (69 mutations), LDA (46 mutations) that interacts with NPC2 to accept cholesterol, and the sterol-sensing domain (SSD) composed of TD3-TD7 (38 mutations). Similarly, the highest gross number of individuals possessing mutations were associated with LDC (383 individuals), LDA (304 individuals), LDI (288 individuals), and CDL (128 individuals), but not the SSD composed of TD3-TD7 (74 individuals). The highest percentage of mutant amino acids per domain were represented by CDN (56.67\%), CDL (55.17\%), TD4 (55.00\%), TD7 (55.00\%), and CDJ (42.86\%) positioned within two of the transmembrane domains of the SSD and the last three cytoplasmic domains for targeting to late-endosomes. To a similar extent, the highest percentage of individuals per domain were represented by CDL (441.38\%), CDN (176.67\%), CDJ (171.43\%), LDC (154.44\%), and TD7 $(155.00 \%)$ positioned within the last three cytoplasmic domains for targeting to late endosomes, the second large luminal domain that binds NPC2 and assorted viruses, and a domain adjacent to the SSD.

Ethnic differences in the prevalence of NPC1 pathogenic mutations. Table 2 summarizes the prevalence of heterozygous and homozygous carriers of NPC1 pathogenic mutations in the six ethnic groups from gnomAD. The coding pathogenic mutations identified in gnomAD are also represented in an NPC1 protein model (Fig. 1). Homozygous/heterozygous compound carriers of partial/complete loss-of-function coding mutations in NPC1 result in NPC1 disease ${ }^{16}$. We identified four homozygous carriers of three rare NPC1 


\begin{tabular}{|c|c|c|c|c|c|c|c|}
\hline & $\begin{array}{l}\text { Global } \\
(\mathrm{N}=122,678)\end{array}$ & African $(\mathrm{N}=8128)$ & $\begin{array}{l}\text { Ashkenazi Jewish } \\
(\mathrm{N}=5040)\end{array}$ & $\begin{array}{l}\text { East Asian } \\
(\mathrm{N}=9197)\end{array}$ & $\begin{array}{l}\text { European } \\
(\mathrm{N}=67,709)\end{array}$ & Latino $(N=17,296)$ & $\begin{array}{l}\text { South Asian } \\
(N=15,308)\end{array}$ \\
\hline${ }^{\mathrm{a} T o t a l}$ alleles, $\mathrm{N}$ & 245,356 & 16,256 & 10,080 & 18,394 & 135,418 & 34,592 & 30,616 \\
\hline${ }^{b}$ Mutated alleles, $N$ & 1574 & 265 & 28 & 107 & 548 & 251 & 375 \\
\hline Frequency, (\%) & $(0.64 \%)$ & $(1.63 \%)$ & $(0.28 \%)$ & $(0.58 \%)$ & $(0.40 \%)$ & $(0.73 \%)$ & $(1.22 \%)$ \\
\hline $\begin{array}{l}\text { Heterozygous car- } \\
\text { riers, } \mathrm{N}\end{array}$ & 1566 & 265 & 28 & 107 & 548 & 251 & 367 \\
\hline Frequency, (\%) & $(1.28 \%)$ & $(3.26 \%)$ & $(0.56 \%)$ & $(1.16 \%)$ & $(0.81 \%)$ & $(1.45 \%)$ & $(2.40 \%)$ \\
\hline $\begin{array}{l}\text { Homozygous car- } \\
\text { ries, } \mathrm{N}\end{array}$ & 4 & 0 & 0 & 0 & 0 & 0 & 4 \\
\hline Frequency, (\%) & $(0.0033 \%)$ & $(0 \%)$ & $(0 \%)$ & $(0 \%)$ & $(0 \%)$ & $(0 \%)$ & $(0.026 \%)$ \\
\hline Sex, M/F & $66,355 / 56,323$ & $3093 / 5035$ & $2590 / 2450$ & $4533 / 4664$ & $37,442 / 30,267$ & $7161 / 10,135$ & $11,536 / 3772$ \\
\hline $\begin{array}{l}\text { Heterozygous carri- } \\
\text { ers, M/F }\end{array}$ & $813 / 753$ & $96 / 169$ & $8 / 20$ & $48 / 49$ & $296 / 252$ & $106 / 145$ & $259 / 108$ \\
\hline $\begin{array}{l}\text { Homozygous carri- } \\
\text { ers, M/F }\end{array}$ & $3 / 1$ & $0 / 0$ & $0 / 0$ & $0 / 0$ & $0 / 0$ & $0 / 0$ & $3 / 1$ \\
\hline \multicolumn{8}{|c|}{ Ethnic-specific mutations } \\
\hline Mutated alleles, $\mathrm{N}$ & 611 & 44 & 7 & 46 & 275 & 107 & 132 \\
\hline $\begin{array}{l}\text { Heterozygous car- } \\
\text { riers, } \mathrm{N}\end{array}$ & 609 & 44 & 7 & 46 & 275 & 107 & 130 \\
\hline $\begin{array}{l}\text { Homozygous carri- } \\
\text { ers, N }\end{array}$ & 1 & 0 & 0 & 0 & 0 & 0 & 1 \\
\hline
\end{tabular}

Table 2. Ethnic differences, sex distribution and global prevalence of pathogenic NPC1 mutations. $M$ male, $F$ female. ${ }^{\text {a }}$ The total number of alleles in each group (determined by multiplying the number of individuals by two). ${ }^{b}$ Pathogenic alleles with a minor allele frequency lower than $1 \%$ in all ethnic groups.

missense mutations (p.Gly149Arg, p.Val780Met, p.Ala1108Thr) out of 122,678 participants (0.0033\%). No homozygous carrier of frameshift, stop gained, essential splice site and start loss mutations was observed in gnomAD. The four homozygous carriers, three males and one female, all belong to the South Asian ethnic group $(\mathrm{N}=15,308$; homozygous carrier frequency: 0.026\%). The other ethnic groups (Ashkenazi Jewish, African, East Asian, European, and Latino) had no homozygous carriers. When the sample was restricted to control individuals, only one homozygous carrier remained in the sample. However, no homozygous carrier was considered as a neurological case in gnom $A D$.

Heterozygous carriers of partial/complete loss-of-function coding mutations in the NPC1 gene confer an increased risk of obesity in East Asian populations ${ }^{14}$. We identified 1566 heterozygous carriers of rare NPC1 mutations out of 122,678 participants (1.28\%). Of the 1566 heterozygous carriers, 28 belong to the Ashkenazi Jewish ethnic group ( $\mathrm{N}=5040$; heterozygous carrier frequency: 0.56\%), 548 to the European ethnic group $(\mathrm{N}=67,709$; heterozygous carrier frequency: 0.81\%), 107 to the East Asian ethnic group ( $\mathrm{N}=9197$; heterozygous carrier frequency: $1.16 \%), 251$ to the Latino ethnic group $(\mathrm{N}=17,296$; heterozygous carrier frequency: $1.45 \%), 367$ to the South Asian ethnic group ( $\mathrm{N}=15,308$; heterozygous carrier frequency: $2.40 \%)$, and 265 to the African ethnic group ( $\mathrm{N}=8128$; heterozygous carrier frequency: 3.26\%). A 5.8-fold difference was observed between the lowest and highest prevalence of heterozygous carriers.

The cumulative frequency of pathogenic mutated alleles in NPC1 was significantly different between the six ethnic groups from gnom $\mathrm{AD}(\mathrm{P}$-value $=0)$, with 5.8-fold difference between the lowest and highest mutation frequency: Ashkenazi Jewish (0.28\%), European (0.40\%), East Asian (0.58\%), Latino (0.73\%), South Asian (1.22\%), and African (1.63\%). Of the 414 mutations, 340 mutations (82\%) are unique to only one ethnic group: five belonging to the Ashkenazi Jewish population, 32 in the African population, 36 in the East Asian population, 43 in the Latino population, 57 in the South Asian population, and 167 mutations unique to the European population (Supplementary Table 1). These ethnic-specific mutations represent 609 heterozygous carriers and one homozygous carrier (p.Ala1108Thr). The distribution of ethnic-specific mutations in heterozygous and homozygous carriers of NPC1 pathogenic mutations is significantly different between the ethnic groups $(\mathrm{P}$-value $=0)$. The cumulative frequency of pathogenic alleles in NPC1 is represented in Fig. 2.

Sex differences in the prevalence of NPC1 pathogenic mutations. Table 2 summarizes male and female distribution of NPC1 pathogenic mutations in heterozygous and homozygous carriers. A significant difference in the prevalence of pathogenic mutations was not observed between male and female individuals globally and within the six ethnic groups. Of the 414 mutations, 149 mutations were male-specific, 143 were femalespecific, and 122 mutations were present in both male and female participants. More details on these mutations can be found in Supplementary Tables 1 and 2 .

\section{Discussion}

Unexpectedly, our findings do not provide evidence for negative selection of LOF, non-synonymous, and synonymous mutations in the NPC1 gene. Further, the pLI value of 0 may be indicative of positive natural selection for LOF mutations, although this conclusion should be interpreted with caution because the prevalence of observed 


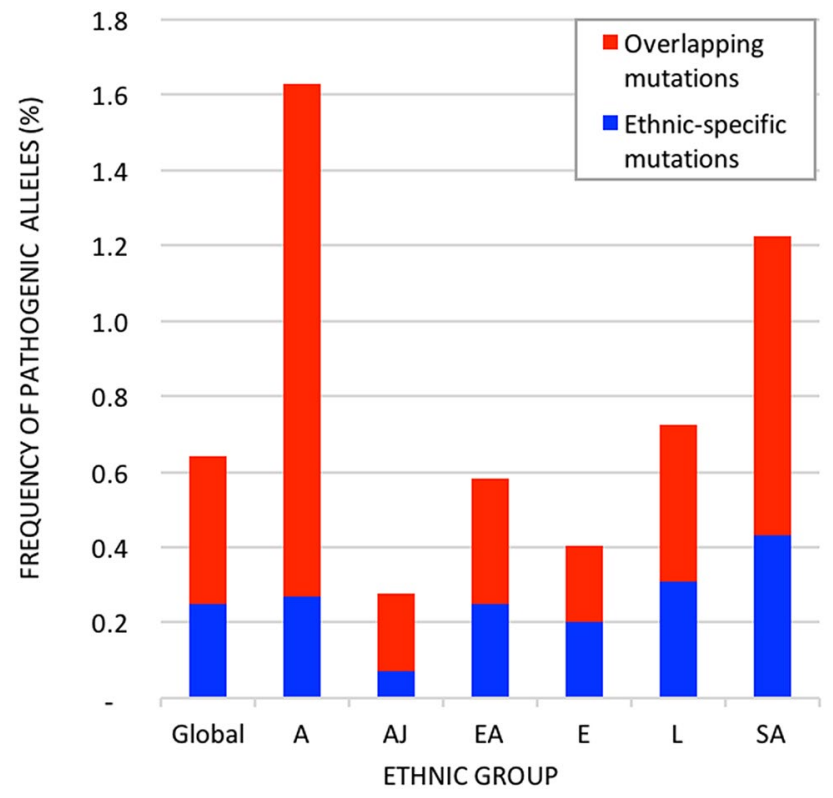

Figure 2. The cumulative frequency of pathogenic mutated alleles in NPC1 globally and by ethnic groups. Ethnic-specific mutations represent mutations that are prevalent in only one ethnic group from gnomAD. Overlapping mutations are prevalent in more than one ethnic group. The global prevalence is described, as well as the prevalence for each of the six populations: Europeans (E), Latino (L), South Asian (SA), East Asian (EA), African/African American (A), and Ashkenazi Jewish (AJ).

LOF mutations was three times lower than the expected value. The length of the gene can influence the reliability of the pLI value where short genes are less likely to be accurately detected ${ }^{32}$, and considering that NPC1 is a longer gene, the 0 value for pLI is likely to be accurate. It may be counterintuitive for LOF mutations in NPC1 to be tolerated, considering that homozygous or heterozygous compound LOF mutations in NPC1 cause a lifethreatening recessive lipid storage disorder ${ }^{16}$. Further, the reproductive fitness of patients with NPC1 disease is substantially compromised due to a shorter life expectancy (10-25 years old), and neurological impairments ${ }^{16}$. The pLI score is more adapted to identify haploinsufficient genes that cause life-threatening dominant diseases. In the case of the NPC1 gene, although a heterozygous mutation does not usually lead development of NPC1 disease, cases have been reported whereby a heterozygous mutation results in partial manifestation of "NPC1 heterozygous disease" 33,34 . Carriers of pathogenic NPC1 mutations have been described to have a negative natural selection considering obesity has been linked to infertility ${ }^{35}$. However, human evolution has occurred during historic periods of famine ${ }^{35}$ when heterozygous mutations may actually positively impact fitness by providing an evolutionary advantage due to an increased ability to store fat, aligning with the 'thrifty genotype hypothesis $^{26,28}$. Therefore, the NPC1 gene may have been subject to balanced selection, where heterozygotes observed a higher reproductive fitness and homozygotes had a lower fitness, resulting in no negative signature of natural selection $^{26,29,30}$. With respect to the 27 NPC1 protein domains, the highest percentage of mutant amino acids and individuals harboring these mutations per domain were consistent with the last three cytoplasmic domains for targeting to late endosomes, the second large luminal domain that binds NPC2, and a domain adjacent to the SSD. Therefore, these NPC1 protein domains, which are of central importance for structure and function are enriched with pathogenic mutations, may prove to be deleterious in the homozygous state and possibly advantageous in the heterozygous state assuming balancing selection.

Our study indicates that the frequency of NPC1 pathogenic mutations differs significantly among ethnic groups, with over $80 \%$ of the mutations found in one ethnic group. In fact, the divergent pattern observed is consistent with a balanced selection model, where mutations with low and relatively equal frequencies among ethnic groups may have been negatively selected against ${ }^{27}$. Conversely, mutations with highly varied frequencies between ethnic groups may be explained by local patterns of positive selection ${ }^{27}$. Liu et al. recently described that East Asian heterozygous NPC1 LOF mutation carriers had a significantly higher BMI and risk of severe obesity than non-carriers ${ }^{14}$. The prevalence of NPC1 heterozygous carriers in our study is consistent with previously reported prevalence of obesity across different ethnic groups. Most notably, African, Latino, and South Asian populations have been reported to have a higher risk of obesity/abdominal obesity than Caucasians and East Asians, which aligns with the present findings where the highest prevalence of deleterious NPC1 mutations was observed in the Latino, South Asian, and African groups ${ }^{36,37}$. NPC1 heterozygous carriers of pathogenic mutations represent $0.56-3.26 \%$ of the gnomAD population depending on the ethnic group. Considering that these individuals have potentially a high risk to develop obesity ${ }^{14}, N P C 1$ may account for a non-negligible fraction of obesity cases in the general population. In fact, four heterozygous NPC1 variants (p.Thr743Ile, p.Arg794Trp, p.Val1044Met, p.Thr1176Met) were found by Liu et al. in seven obese young patients with BMIs ranging from 35.4 to 45.4 , and were also captured in our sample ${ }^{14}$. In addition, Lui et al. describe a heterozygous LOF NPC1 gene 
mutation (p.Val780fs ${ }^{\star}$ ) in an obese patient with a BMI of $45.6^{14}$, suggesting that LOF NPC1 mutations may indeed predispose individuals to a higher BMI. Although Lie et al. report sex-specific associations between pathogenic NPC1 mutations and obesity, we found no significant difference between the prevalence of mutations in males and females. It is important to note that the study by Lui et al. was limited to a modest East Asian sample and further genotype-phenotype studies are required confirm associations with obesity in diverse ethnic groups.

We identified four homozygous carriers of three rare NPC1 missense mutations out of 122,678 participants $(0.0033 \%)$, all belonging to the South Asian ethnic group. It is possible that these individuals are in fact affected by NPC1 disease, known to be caused by homozygous or heterozygous compound NPC1 pathogenic mutations. The prevalence of homozygous carriers in the current population $(0.0033 \%)$ is consistent with the estimated prevalence of Niemann-Pick disease type C on ORPHANET (0.001-0.009\%), keeping in mind that NPC1 mutations account for $95 \%$ of disease cases ${ }^{16}$. Further, endogamy has been postulated to underlie a high recessive disease burden in South Asian populations ${ }^{38}$. The ancestral variants are non-polar and shielded from the aqueous environment due to being buried within the hydrophobic core of LDA (p.Gly149Arg) and associated with alpha helices TD7 (p.Val780Met) and TD9 (p.Ala1108Thr) within the hydrophobic core of a phospholipid bilayer. Moreover, compared to the ancestral variants, the respective derived variants possess side chains with a much larger spatial area. Therefore, since the combined homozygous mutations have different physiochemical properties, the protein secondary and tertiary structures will be adversely effected, which in turn will negatively influence thermodynamic stability resulting in a biological loss-of-function. Our findings suggest that this increased risk may also extend to NPC1 disease. To our knowledge, NPC1 disease has yet to be studied in South Asian populations. This may represent a priority for future investigations to increase our understanding of the populations at greatest risk of developing NPC1 disease. Considering that the gnomAD database attempts to exclude cases of rare pediatric life-threatening disease, our finding of four potential NPC1 cases may have several plausible explanations. These individuals may have experienced a late onset or non-fully penetrant disease manifestations which did not meet the criteria for a pediatric life-threatening disease or these cases may have simply not been captured by gnomAD. Notably, variant p.Gly149Arg has been reported as benign/likely benign for NPC1 disease in ClinVar, although clinical features of patients with the variant were not described. As for carriers of NPC1 disease, it is possible that this variant is only partially deleterious and thereby placing patients at increased risk of less penetrant disease manifestations such as obesity. These findings should be interpreted cautiously, as a conclusion cannot be made without clinical and in vitro functional characterization data confirming that these mutations (p.Gly149Arg, p.Val780Met, p.Ala1108Thr) are indeed disease causing.

The current investigation has several strengths. This is the first study to investigate the prevalence of NPC1 mutations in diverse ethnic groups, providing a global understanding of the implications of NPC1 in obesity risk and NPC1 disease. This is also the first investigation of a potential signature of natural selection for the NPC1 gene. Further, conclusions are drawn from a large sample size of 122,678 participants from gnomAD, reducing bias associated with random sampling error and small sample size. This study also presents several limitations. The sample population on gnomAD is a mixture of the general population along with disease cases (e.g., type 2 diabetes, cancer, neurological conditions), therefore limiting the applicability of the current findings to the general population. The data on gnomAD is aggregated from multiple sources and the sampling methods of each study is unavailable, introducing potential bias in the population-specific results. In addition, gnomAD does not represent all ethnic groups, such as Arabs, Pacific Islanders, and Native Australians. Phenotypic data is also not accessible making it difficult to ascertain the presence and severity of disease associated with specific mutations. The pathogenicity of missense mutations was investigated using in silico prediction tools and the ClinVar database. We used SIFT and Polyphen 2 as these were available in gnomAD and enabled large-scale, transparent, and reproducible findings, although we acknowledge that other in silico tools have been described with better prediction values for cholesterol transport proteins ${ }^{39}$. We are also aware that in vitro experiments will have enhanced the functional characterization of mutations. We also excluded NPC1 coding synonymous mutations based on their low likelihood of pathogenicity, but we acknowledge that some of these mutations may have deleterious consequences on NPC1 expression/function ${ }^{40}$. Finally, the functionalities of the gnomAD database do not enable the identification of heterozygous compound mutations, and individuals affected by severe pediatric diseases have been removed, which may have resulted in an underestimation of the prevalence of suspected NPC1 disease cases.

In summary, we did not identify a negative selection for pathogenic mutations in the NPC1 gene. This result is consistent with a balanced selection model, where NPC1 heterozygotes display a higher reproductive fitness and homozygotes have a lower fitness. Our study shows that the frequency of NPC1 pathogenic mutations differs significantly among ethnic groups. Depending on the ethnic group, between $0.56 \%$ and $3.26 \%$ of the gnomAD population is heterozygous for NPC1 pathogenic mutations that may result in a high-risk of obesity. In addition, homozygous carriers of NPC1 pathogenic mutations that can result in NPC1 disease were only found in the South Asian population $(0.026 \%)$. Our study contributes to a better understanding of the global impact of NPC1 mutations in human diseases.

\section{Subjects and methods}

Participants. The dataset on gnomAD v2.1.1 includes 141,456 unrelated individuals (125,748 exomes and 15,708 genomes) sequenced to perform population-genetic and disease-specific studies. Of these individuals, sub-samples were defined by gnomAD: 60,146 samples as controls (not a case in a case/control study of common disease), 134,187 samples as non-cancer (not a case in a cancer study), 114,704 samples as non-neuro (not a case in a case/control study of neurological disease). Individuals affected by severe pediatric diseases were removed. We chose to only include the exome data of the complete gnomad v2.1.1 dataset in our analysis to keep the sample size stable for estimating the prevalence of mutations. Exome sequenced participants in gnomAD 
have been grouped in seven ethnic groups using a principal component analysis to distinguish the major axes of geographic ancestry: Europeans $(\mathrm{N}=67,709)$, Latino $(\mathrm{N}=17,296)$, South Asians $(\mathrm{N}=15,308)$, East Asians $(\mathrm{N}=9197)$, Africans/African Americans $(\mathrm{N}=8128)$, Ashkenazi Jewish $(\mathrm{N}=5040)$ and other $(\mathrm{N}=3070)$. As the category 'other' is comprised of several ethnicities and does not provide details on the geographic origin of individuals, we did not include them in our analyses. Additional details regarding the sample populations have been described previously ${ }^{32,41}$. The data was collected from gnomAD on April 17th, 2020. Research was conducted in accordance with relevant guidelines and regulations of the Declaration of Helsinki and was approved by all the local institutional review boards of studies participating to ExAC and gnomAD (e.g. Broad Institute), as previously described ${ }^{32,41}$. Written informed consent was obtained from each subject prior to participation.

Sequencing data quality control, release, and reporting in ExAC and gnomAD. Full details of data processing, variant calling, filtering process and variant annotation in ExAC have been previously described ${ }^{32}$. GnomAD was quality controlled and analyzed using the Hail open source framework (https://githu b.com/hail-is/hail) ${ }^{41}$. This data set can be accessed via the gnomAD Browser (https://gnomad.broadinstitute. $\operatorname{org} /)$.

Functional characterization of NPC1 coding mutations. Isoform I of the NPC1 protein is the canonical sequence and therefore all reported protein consequences in the manuscript correspond to isoform I. We only included NPC1 mutations that displayed a minor allele frequency lower than $1 \%$ in all ethnic groups. Nonsense, splice acceptor, splice donor, and frameshift mutations were considered as LOF. Whether non-synonymous mutations significantly affected protein function was investigated by the SIFT and PolyPhen2 software. We previously showed that Polyphen 2 predictions are comparable to the conclusions resulting from in vitro tests for the PCSK1 gene ${ }^{42}$. We also included NPC1 coding mutations reported as pathogenic/likely pathogenic in the ClinVar database ${ }^{43}$. Synonymous coding mutations were not considered pathogenic. The current parameters in gnom $\mathrm{AD}$ that were used to assess pathogenicity were not available for in frame insertions/deletions so these mutations were excluded from further study. Mutations flagged by gnomAD as dubious variant annotation or quality were not considered. Pathogenic mutations present in the 'other' ethnicity group and mutations with protein consequences not corresponding to isoform 1 were also excluded.

Evolutionary genetic analyses. Lek et al. has established the probability of each gene being LOF (nonsense and essential splice site) intolerant (pLI) using the expectation-maximization (EM) algorithm in ExAC ${ }^{32}$. A $p L I \leq 0.1$ indicates that the gene is LOF tolerant, meaning there is no substantial negative selection against loss-of-function mutations. A pLI $\geq 0.9$ indicates that the gene is LOF intolerant. Lek et al. created $\mathrm{Z}$ scores to establish the significance of the deviation of observed synonymous and non-synonymous variant counts per gene from expectation. Significantly elevated synonymous and non-synonymous counts corresponded to $Z$ scores $<-3.71$ and $<--3.09$, respectively. Significantly depleted synonymous and non-synonymous counts corresponded to $\mathrm{Z}$ scores $>3.71$ and $>3.09$, respectively. These $\mathrm{Z}$-score values are equivalent to a P-value of $10^{-3}$ and represent the significance threshold when splitting transcripts into constrained and unconstrained classes ${ }^{32}$. According to the gnom $\mathrm{AD}$ browser information, an observed/expected mutation ratio $90 \%$ confidence interval upper bound value $<0.35$ is indicative of strong mutation intolerance.

Statistical analyses. The comparison of pathogenic mutation frequencies in NPC1 and sex distributions among ethnic groups in gnom $A D$ was conducted using Chi-square tests. All reported $P$-values are two-sided. $P$-values of less than 0.05 were considered significant.

Compliance with ethical standards. Written informed consent was obtained from each participant before participation, in accordance with the Declaration of Helsinki.

\section{Data availability}

The data that supports this investigation are publicly and openly available in the Genome Aggregation Database V 2.1.1 at https://gnomad.broadinstitute.org/.

Received: 13 May 2020; Accepted: 21 September 2020

Published online: 02 November 2020

\section{References}

1. Abarca-Gómez, L. et al. Worldwide trends in body-mass index, underweight, overweight, and obesity from 1975 to 2016 : A pooled analysis of 2416 population-based measurement studies in 128.9 million children, adolescents, and adults. Lancet 390, 2627-2642 (2017).

2. Guh, D. P. et al. The incidence of co-morbidities related to obesity and overweight: A systematic review and meta-analysis. $B M C$ Public Health 9, 88. https://doi.org/10.1186/1471-2458-9-88 (2009).

3. Yusuf, S. et al. Obesity and the risk of myocardial infarction in 27,000 participants from 52 countries: A case-control study. Lancet 366, 1640-1649. https://doi.org/10.1016/S0140-6736(05)67663-5 (2005).

4. Arnold, M. et al. Global burden of cancer attributable to high body-mass index in 2012: A population-based study. Lancet Oncol. 16, 36-46. https://doi.org/10.1016/S1470-2045(14)71123-4 (2015).

5. Members, E. P. et al. Executive summary: Guidelines (2013) for the management of overweight and obesity in adults. Obesity (Silver Spring) 22, S5-S39 (2014).

6. Pigeyre, M., Yazdi, F. T., Kaur, Y. \& Meyre, D. Recent progress in genetics, epigenetics and metagenomics unveils the pathophysiology of human obesity. Clin. Sci. (Lond.) 130, 943-986. https://doi.org/10.1042/CS20160136 (2016). 
7. Alyass, A., Turcotte, M. \& Meyre, D. From big data analysis to personalized medicine for all: Challenges and opportunities. $B M C$ Med. Genom. 8, 33. https://doi.org/10.1186/s12920-015-0108-y (2015).

8. Reddon, H., Gueant, J. L. \& Meyre, D. The importance of gene-environment interactions in human obesity. Clin. Sci. (Lond.) 130, 1571-1597. https://doi.org/10.1042/CS20160221 (2016).

9. Stryjecki, C., Alyass, A. \& Meyre, D. Ethnic and population differences in the genetic predisposition to human obesity. Obes. Rev. 19, 62-80. https://doi.org/10.1111/obr.12604 (2018).

10. Yang, J. et al. Genetic variance estimation with imputed variants finds negligible missing heritability for human height and body mass index. Nat. Genet. 47, 1114-1120. https://doi.org/10.1038/ng.3390 (2015).

11. Kaur, Y., de Souza, R. J., Gibson, W. T. \& Meyre, D. A systematic review of genetic syndromes with obesity. Obes. Rev. 18, 603-634. https://doi.org/10.1111/obr.12531 (2017).

12. Pigeyre, M. \& Meyre, D. Monogenic obesity. Pediatric Obesity: Etiology, Pathogenesis and Treatment, 2nd edn., (ed Freemark, M.) 135-152 (Humana Press, Totowa, 2018).

13. Meyre, D. et al. Genome-wide association study for early-onset and morbid adult obesity identifies three new risk loci in European populations. Nat. Genet. 41, 157-159. https://doi.org/10.1038/ng.301 (2009).

14. Liu, R. et al. Rare loss-of-function variants in NPC1 predispose to human obesity. Diabetes 66, 935-947. https://doi.org/10.2337/ db16-0877 (2017).

15. Lamri, A., Pigeyre, M., Garver, W. S. \& Meyre, D. The extending spectrum of NPC1-related human disorders: From Niemann-Pick C1 Disease to obesity. Endocr. Rev. https://doi.org/10.1210/er.2017-00176 (2018).

16. Vanier, M. T. Niemann-Pick disease type C. Orphanet. J. Rare Dis. 5, 16. https://doi.org/10.1186/1750-1172-5-16 (2010).

17. Wassif, C. A. et al. High incidence of unrecognized visceral/neurological late-onset Niemann-Pick disease, type C1, predicted by analysis of massively parallel sequencing data sets. Genet. Med. 18, 41-48. https://doi.org/10.1038/gim.2015.25 (2016).

18. Lopez, M. E., Klein, A. D., Dimbil, U. J. \& Scott, M. P. Anatomically defined neuron-based rescue of neurodegenerative NiemannPick type C disorder. J. Neurosci. 31, 4367-4378. https://doi.org/10.1523/JNEUROSCI.5981-10.2011 (2011).

19. Bosch, M. et al. Hepatic primary and secondary cholesterol deposition and damage in Niemann-Pick disease. Am. J. Pathol. 186, 517-523. https://doi.org/10.1016/j.ajpath.2015.12.002 (2016).

20. Yu, T., Shakkottai, V. G., Chung, C. \& Lieberman, A. P. Temporal and cell-specific deletion establishes that neuronal Npc1 deficiency is sufficient to mediate neurodegeneration. Hum. Mol. Genet. 20, 4440-4451. https://doi.org/10.1093/hmg/ddr372 (2011).

21. Turcot, V. et al. Protein-altering variants associated with body mass index implicate pathways that control energy intake and expenditure in obesity. Nat. Genet. 50, 26-41. https://doi.org/10.1038/s41588-017-0011-x (2018).

22. Jelinek, D., Heidenreich, R. A., Erickson, R. P. \& Garver, W. S. Decreased Npc1 gene dosage in mice is associated with weight gain. Obesity (Silver Spring) 18, 1457-1459. https://doi.org/10.1038/oby.2009.415 (2010).

23. Castillo, J. J. et al. The Niemann-Pick C1 gene interacts with a high-fat diet to promote weight gain through differential regulation of central energy metabolism pathways. Am. J. Physiol. Endocrinol. Metab. https://doi.org/10.1152/ajpendo.00369.2016 (2017).

24. Jelinek, D. et al. Npc1 haploinsufficiency promotes weight gain and metabolic features associated with insulin resistance. Hum. Mol. Genet. 20, 312-321. https://doi.org/10.1093/hmg/ddq466 (2011).

25. Jelinek, D., Castillo, J. J., Heidenreich, R. A. \& Garver, W. S. The C57BL/6J Niemann-Pick C1 mouse model with decreased gene dosage is susceptible to increased weight gain when fed a high-fat diet: Confirmation of a gene-diet interaction. Gene 568, 112-113. https://doi.org/10.1016/j.gene.2015.05.025 (2015).

26. Qasim, A. et al. On the origin of obesity: Identifying the biological, environmental and cultural drivers of genetic risk among human populations. Obes. Rev. 19, 121-149. https://doi.org/10.1111/obr.12625 (2018).

27. Mitchell-Olds, T., Willis, J. H. \& Goldstein, D. B. Which evolutionary processes influence natural genetic variation for phenotypic traits?. Nat. Rev. Genet. 8, 845-856. https://doi.org/10.1038/nrg2207 (2007).

28. Neel, J. V. Diabetes mellitus: A "thrifty" genotype rendered detrimental by "progress"?. Am. J. Hum. Genet. 14, 353-362 (1962).

29. Weatherall, D., Akinyanju, O., Fucharoen, S., Olivieri, N. \& Musgrove, P. In Disease Control Priorities in Developing Countries (eds Jamison, D.T., Breman, J.G., \& Measham, A.R.) Ch. 34, 663-680 (Oxford University Press, Oxford, 2006).

30. Allison, A. C. Polymorphism and natural selection in human populations. Cold Spring. Harb. Symp. Quant. Biol. 29, 137-149 (1964).

31. Davies, J. P., Chen, F. W. \& Ioannou, Y. A. Transmembrane molecular pump activity of Niemann-Pick C1 protein. Science 290, 2295-2298. https://doi.org/10.1126/science.290.5500.2295 (2000).

32. Lek, M. et al. Analysis of protein-coding genetic variation in 60,706 humans. Nature 536, 285-291. https://doi.org/10.1038/natur e19057 (2016).

33. Josephs, K. A., Matsumoto, J. Y. \& Lindor, N. M. Heterozygous Niemann-Pick disease type C presenting with tremor. Neurology 63, 2189-2190 (2004).

34. Harzer, K., Beck-Wodl, S. \& Bauer, P. Niemann-pick disease type C: New aspects in a long published family-partial manifestations in heterozygotes. JIMD Rep. 12, 25-29. https://doi.org/10.1007/8904_2013_240 (2014).

35. Erickson, R. P. Do GWAS and studies of heterozygotes for NPC1 and/or NPC2 explain why NPC disease cases are so rare?. J. Appl. Genet. 59, 441-447 (2018).

36. Misra, A. \& Khurana, L. Obesity-related non-communicable diseases: South Asians vs White Caucasians. Int. J. Obes. 35, 167-187 (2011).

37. Ogden, C. L., Carroll, M. D., Fryar, C. D. \& Flegal, K. M. Prevalence of obesity among adults and youth: United States, 2011-2014. NCHS Data Brief 219, 1-8 (2015).

38. Nakatsuka, N. et al. The promise of discovering population-specific disease-associated genes in South Asia. Nat. Genet. 49, 14031407 (2017).

39. López de Frutos, L., Cebolla, J. J., Irún, P., Köhler, R. \& Giraldo, P. Web-based bioinformatics predictors: Recommendations to assess lysosomal cholesterol trafficking diseases-related genes. Methods Inf. Med. 58, 50-59 (2019).

40. Braun, T. A. et al. Non-exomic and synonymous variants in ABCA4 are an important cause of Stargardt disease. Hum. Mol. Genet. 22, 5136-5145. https://doi.org/10.1093/hmg/ddt367 (2013).

41. Karczewski, K. J. et al. Variation across 141,456 human exomes and genomes reveals the spectrum of loss-of-function intolerance across human protein-coding genes. bioRxiv https://doi.org/10.1101/531210 (2019).

42. Creemers, J. W. et al. Heterozygous mutations causing partial prohormone convertase 1 deficiency contribute to human obesity. Diabetes 61, 383-390. https://doi.org/10.2337/db11-0305 (2012).

43. Landrum, M. J. et al. ClinVar: Public archive of interpretations of clinically relevant variants. Nucleic Acids Res. 44, D862-868. https://doi.org/10.1093/nar/gkv1222 (2016).

\section{Acknowledgements}

We would like to thank all the participants of the study. We also thank Regan Huynh for her technical assistance. The authors would like to thank the Exome Aggregation Consortium (ExAC), the Genome Aggregation Database (gnomAD), and the groups that provided exome and genome variant data to these resources ${ }^{32,41}$. David Meyre 
holds a Tier 2 Canada Research Chair in Genetics of Obesity. The funding agency was not involved in the design, conduct, or publication of the study.

\section{Author contributions}

A.C., W.S.G. and D.M. contributed to study conception, data analysis, data interpretation, and writing and revising the manuscript.

\section{Competing interests}

The authors declare no competing interests.

\section{Additional information}

Supplementary information is available for this paper at https://doi.org/10.1038/s41598-020-75919-4.

Correspondence and requests for materials should be addressed to D.M.

Reprints and permissions information is available at www.nature.com/reprints.

Publisher's note Springer Nature remains neutral with regard to jurisdictional claims in published maps and institutional affiliations.

(c) (i) Open Access This article is licensed under a Creative Commons Attribution 4.0 International License, which permits use, sharing, adaptation, distribution and reproduction in any medium or format, as long as you give appropriate credit to the original author(s) and the source, provide a link to the Creative Commons licence, and indicate if changes were made. The images or other third party material in this article are included in the article's Creative Commons licence, unless indicated otherwise in a credit line to the material. If material is not included in the article's Creative Commons licence and your intended use is not permitted by statutory regulation or exceeds the permitted use, you will need to obtain permission directly from the copyright holder. To view a copy of this licence, visit http://creativecommons.org/licenses/by/4.0/.

(c) The Author(s) 2020 